\title{
Research and Application on the Third Party Quality Monitoring for Social Public Service: A Case Study of Survey Data in Liaoning Province
}

\author{
Liao Jingxing ${ }^{1, a}$, Feng Lei ${ }^{2, b}$, Liu Siyue ${ }^{3, c}$ \\ ${ }^{1}$ China National Institute of Standardization Beijing, China \\ ${ }^{2}$ China National Institute of Standardization Beijing, China \\ ${ }^{3}$ China National Institute of Standardization Beijing, China
}

\begin{abstract}
To monitor study and analyze the social public service quality is conducive to promote the construction of a service-oriented government that is satisfactory to the people. In this paper, third-party quality monitoring was conducted in 14 cities of Liaoning Province from 11 public service fields such as living environment, public transportation, infrastructure, medical and health care, public security, culture and sports, compulsory education, pension service, employment service, social security and administrative convenience. The results showed that the public's satisfaction with social security, compulsory education, living environment, public security and employment services was low, which needed to be paid attention to and further improved by Liaoning Provincial Government.
\end{abstract}

\section{Preface}

To improve the quality of public service is an inevitable requirement of social development in the new era. To meet the people's needs for a better life, it is necessary to provide high-quality public service for the people. It was clearly stated in the report of the $19^{\text {th }}$ National Congress of the Communist Party of China that "The public service system should be improved to ensure the basic life of the masses and continuously meet the growing needs of the people for a better life"[1]. To improve the public service quality and enhance the satisfaction and well-being of the masses with high-quality services are urgent requirements put forward by the Party and the people for the public service function of the government in the new era. Therefore, it is of great theoretical and practical significance to monitor the quality of social public service, objectively evaluate the public sector's selfefficacy and meet the public demand, and analyze the causes of the gap, so as to promote the construction of service-oriented government with people's satisfaction[2]

\section{Content And Form of the ThiRd PARTy QUALITY MONITORING}

In accordance with the industrial planning priorities of the Outline for Quality Development (2011-2020), the "Twelfth Five-Year Plan" for the National Basic Public Service System and the requirements of the Technical Guidelines for the Monitoring of Urban Public Service Quality, and in combination with the economic and social development characteristics of Liaoning Province, 11 public service fields including living environment, public transportation, infrastructure, medical and health, public security, culture and sports, compulsory education, pension service, employment service, social security, and administrative convenience were monitored[3].

In order to obtain more objective and fair data, in the third party quality monitoring, stratified sampling was adopted, the form of questionnaire was used, the face-toface interviews on the streets were carried out, covering 14 cities including Shenyang, Dalian, Anshan, Chaoyang, Jinzhou, Tieling, Huludao, Liaoyang, Fuxin, Fushun, Yingkou, Benxi, Dandong and Panjin. According to the relevant requirements and methods for statistical survey sampling, a total of 3,600 valid samples from 11 public service areas were collected from the monitoring of social public service quality in Liaoning Province in 2019[4]. The number and proportion of samples in each city are shown in Figure 1. 

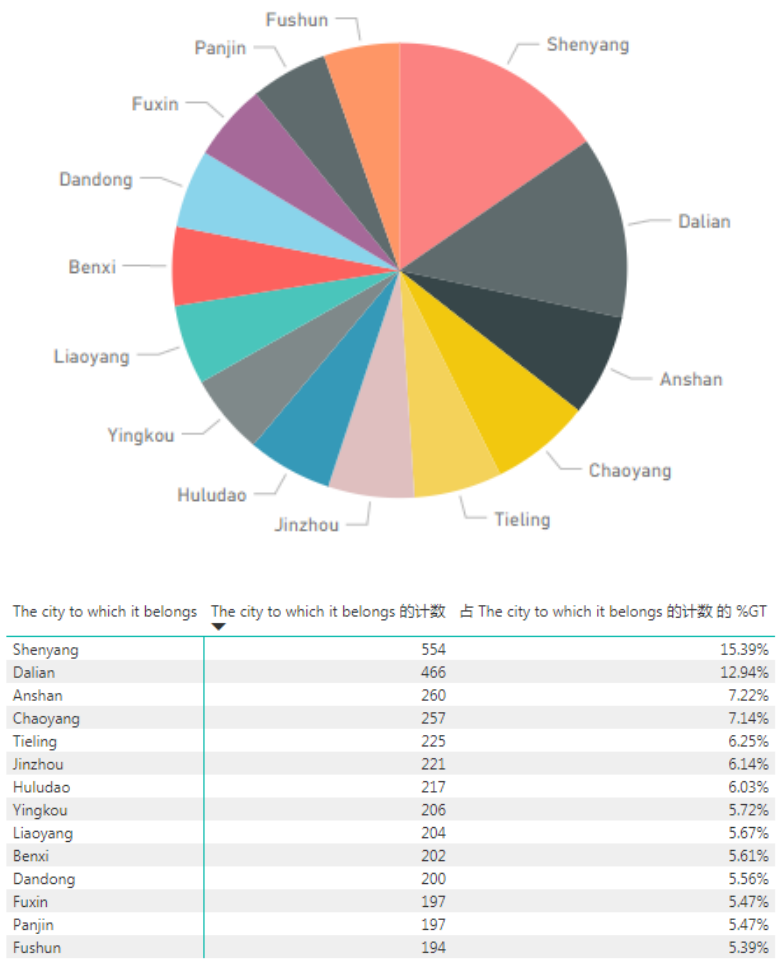

Figure 1. Number of samples and proportion in each city

\section{The Results of Third Party Quality MONITORING}

In 2019, the quality of public service in Liaoning Province was at a "relatively satisfactory" level[5], with a monitoring score of 67.23 points, a slight decrease of 0.56 points compared with the previous year, and the overall outcome comparison are shown in Figure 2.

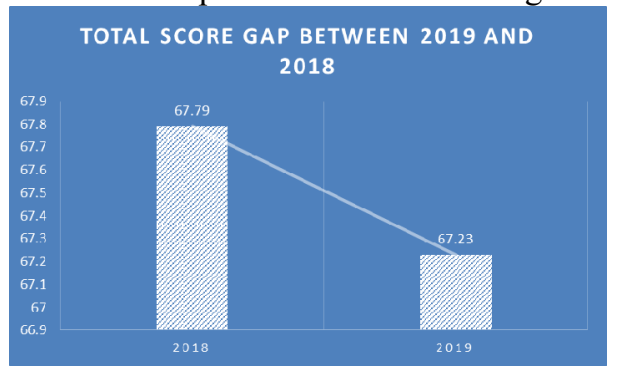

Figure 2. Overall Comparison of Public Service Quality Monitoring in Liaoning Province

In 2019, the satisfaction scores of 11 areas of public service quality monitoring in Liaoning Province ranged from high to low, in the following order: public transportation, with a score of 69.71 points, medical and health, with a score of 68.24 points, pension service, with a score of 67.92 points, administrative convenience, with a score of 67.77 points, cultural and sports, with a score of 67.63 points, infrastructure, with a score of 67.32 points, social security, with a score of 66.78 points, compulsory education, with a score of 66.66 points, living environment, with a score of 66.51 points, public security, with a score of 66.06 points, and the employment service, with a score of 64.91 points. Among them, the score of public transportation was the highest and the score of employment services was the lowest. The first six first-level indicators were higher than the overall score of public service quality monitoring in Liaoning Province, and the last 5 first-level indicators were lower than the overall score of public service quality monitoring in Liaoning Province. A comparison of the data is shown in Figure 3.

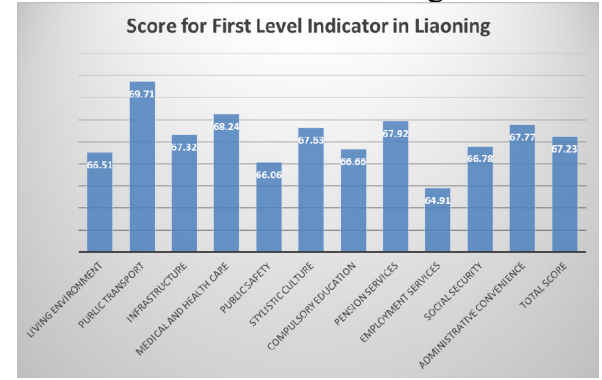

Figure 3. Public satisfaction with the level 1 indicator for public service

\section{Analysis of Influencing factors of Public Service QuALITY}

The monitoring results showed that public satisfaction with service in the five areas of social security, compulsory education, living environment, public security and employment service was low[6]. Through a further analysis of the causes for low social satisfaction in these five areas, the main factors affecting the low satisfaction in various public service indicators can be obtained[7].

\subsection{Employment Services}

In the public service monitoring in Liaoning province in 2019, the satisfaction score of employment services was the lowest, 64.91 points, which was 2.32 points lower than the overall level of public service in Liaoning province. The second-level monitoring indicator under the first-level indicator of employment services included "employment information release", "employment assistance" and "overall feeling of employment services", with scores of 65.62 points, 64.67 points and 63.82 points, respectively.

Through further follow-up and survey for dissatisfaction with employment services, it was found that $45 \%$ of the dissatisfied public reported fewer channels of consultation; $26 \%$ of the dissatisfied public reported that employment information was not released in a timely manner; and $16 \%$ of the dissatisfied public reported that the quality of job fairs was poor. The proportional annular figure $2-3$ is shown by the annular figure, few consultation channels, release of employment behind time and poor quality of job fairs are the main factors that make the public dissatisfied with the quality of employment services[8], as shown in Figure 4. 


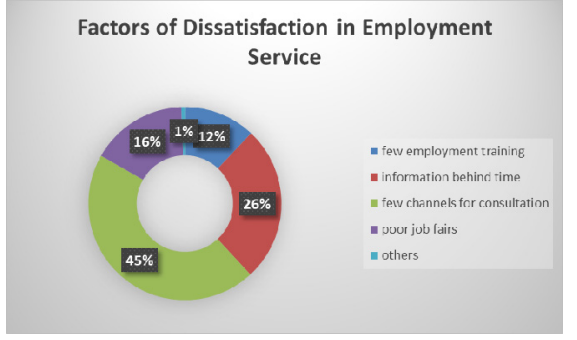

Figure 4. Reasons for Public Dissatisfaction with Employment Services

\subsection{Public Security Services}

The public security service scored 66.06 points, ranking second from the bottom, 1.17 points lower than the overall level of public service in Liaoning Province. The second level monitoring indicator under the first level indicator of public security include "education and publicity of public safety knowledge", "urban public security maintenance level", "overall feeling of public security situation" and "emergency treatment", with scores of 66.58 points, 66.02 points, 65.74 points and 65.25 points, respectively.

Through further survey of the dissatisfied with public security, it was found that $46 \%$ of the dissatisfied public reflected "network insecurity", 27\% of the dissatisfied public reflected "personal safety was threatened", and $15 \%$ of the dissatisfied public reflected the existence of "traffic insecurity", which showed that the above three reasons were the main factors affecting public dissatisfaction with public security, as shown in Figure 5.

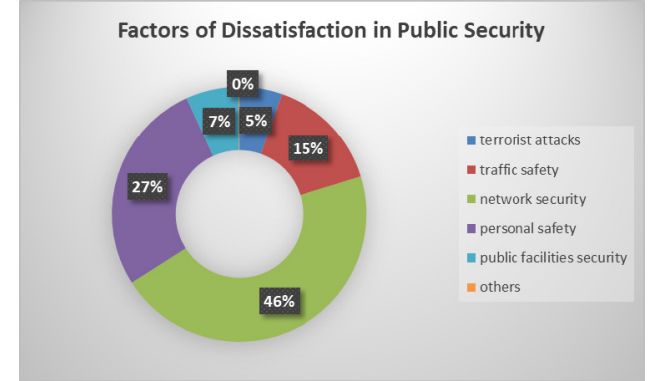

Figure 5. Reasons for Public Dissatisfaction with Public Security

\subsection{Living Environment Service}

The monitoring result of environmental governance service quality was 66.51 points, which was 0.72 points lower than the overall level of public service in Liaoning Province. The second level monitoring indicators under first level indicator of living environment service covered urban afforestation, atmosphere, noise pollution control and the overall feeling of living environment, and the scores was 66.48 points, 66.44 points and 66.17 points, respectively.

Through the further analysis on the reasons for public dissatisfaction with living environmental services, it was found that $31 \%$ of the dissatisfied public reflected poor urban afforestation, $28 \%$ of the dissatisfied public reflected serious air pollution, and $17 \%$ of the dissatisfied public reflected serious river pollution, which indicated that the three above-mentioned indicators were the main factors for public dissatisfaction with living environmental service, as shown in Figure 6.

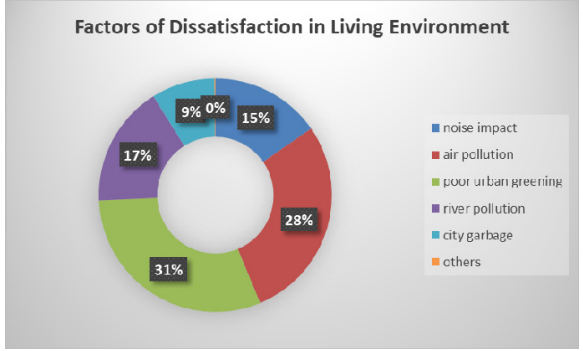

Figure 6. Reasons for Public Dissatisfaction with the Living Environment

\subsection{Compulsory Education Service}

The score of compulsory education service monitoring was 66.66 points, which was 0.57 points lower than the overall level of public service in Liaoning Province. The second level monitoring indicator under first level indicator of compulsory education service covered the feeling of compulsory education service quality, campus safety management and the strength of school teachers, the scores were 66.94 points, 66.75 points and 65.82 points respectively.

Through the further analysis of the dissatisfied public of compulsory education services, it was found that $41 \%$ of the dissatisfied public reflected the existence of campus violence, $33 \%$ of the dissatisfied public reflected the existence of large differences in urban and rural education, and $20 \%$ of the dissatisfied public reflected the heavy burden of school work. Thus, the above three indicators were the main factors for the public to feel dissatisfied with the compulsory education service, as shown in Figure 7.

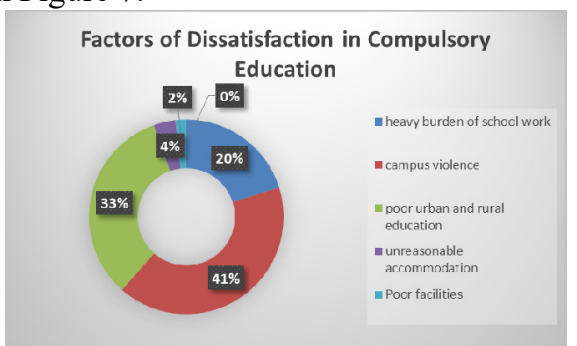

Figure 7. Reasons for Public Dissatisfaction with Compulsory Education Service

\subsection{Social Security Service}

The public satisfaction score of social security service was 66.78 points, which was 0.45 points lower than the overall level of public service in Liaoning Province. The second level monitoring indicator under the first level indicator of social security service covered "the overall feeling of social security service", "the efficiency of social security service" and "the content of social security service". The scores were 67.30 points, 66.15 points and 65.36 points respectively.

The monitoring results showed that $58 \%$ of the public who were not satisfied with the quality of social security service reflected the narrow coverage of medical insurance, $25 \%$ reflected the poor social security 
connection, and $15 \%$ reflected the imbalance of the pension treatment, thus the above-mentioned three indicators were the main factors that the public was not satisfied with the social security service, as shown in Figure 8.

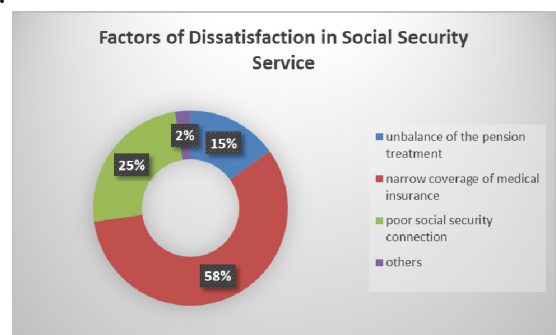

Figure 8. Reasons for Public Dissatisfaction with Social Security Services

\section{Analysis on Social Public Service QUALITY MONITORING GROUP}

\subsection{Monitoring of Social Public Service Quality at Different Age Groups}

The survey results showed that there was a small magnitude of difference in satisfaction with public service among different age groups. The youth group (age group of 18 44 years old) had the highest degree of satisfaction with public services, with a score of 67.57 points, which was higher than the overall level of satisfaction with public service in Liaoning Province; the middle-aged group (age group of 45 59 years old) had the intermediate level of satisfaction with public services, with a score of 66.95 points; the elderly group (age group of $60 \sim 69$ years old) had the lower level of satisfaction with public services, with a score of 66.78 points; and the evaluation of satisfaction with public services in both the middle-aged group and the elderly group was lower than the overall level of public service satisfaction in Liaoning Province. Specific to the first level indicator, in this public service quality monitoring survey, youth group' indicators scores in the living environment, public transportation, infrastructure, medical and health care were higher than another groups. Youth group' indicators scores in the public security, social security and administrative convenience were higher than middleaged group and elderly group. In terms of indicators in culture and sports, pension service and employment service, the scores of elderly group were higher than young and middle-aged groups. And in terms of indicator in compulsory education, the score of middle-aged group was higher than the youth group and the elderly group, the data was shown in Figure 9.

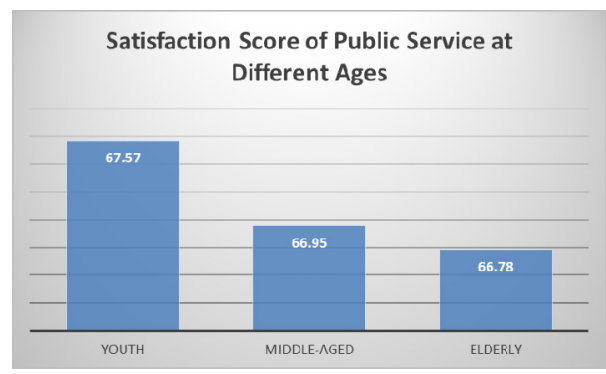

Figure 9. Public Satisfaction with Public Services at Different Ages

\subsection{Monitoring of Social Public Service Quality by Different Gender Groups}

The survey results showed that among the respondents who revealed gender, women were more satisfied with public services than men, with 67.48 points for women and 67.40 points for men, both of which were higher than the overall level of public services in Liaoning Province. Among the first level indicator, women had higher scores than men in the indicators of living environment, infrastructure, medical and health care, public security, compulsory education, social security and administrative convenience for the public service quality monitoring, and men had higher scores than women in the indicators of public transportation, culture and sports, pension service, and employment service. The difference between men and women in the field of public service reflected a relatively strong gender color. With the improvement of women's status in the family and society, women's participation in family affairs and social affairs is also higher, therefore, the right to speak of public service is also increasing, while men enjoy more participation rights and voice in the fields of transportation, sports, employment and so on. The satisfaction evaluation score is shown in Figure 10.

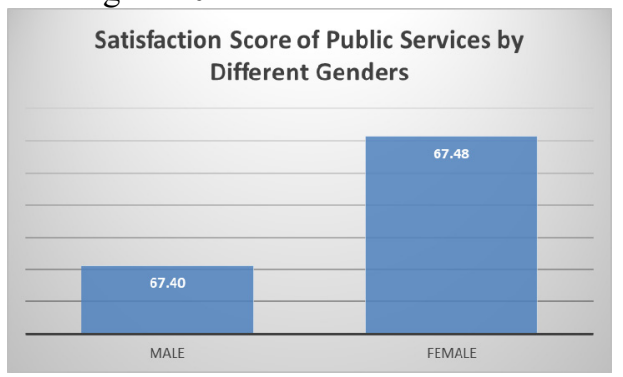

Figure 10. Public Satisfaction with Public Services of Different Genders

\subsection{Monitoring of Social Public Service Quality of Groups by Different Education Backgrounds}

There were significant differences in the satisfaction of public service among the groups with different education levels, among which the satisfaction scores of groups with master's degree or above, college bachelor's degree, and college degree were higher than the overall level of public service satisfaction in Liaoning Province, followed by 70.94 points, 68.49 points and 68.01points respectively, the scores of groups with junior high school education, high school/secondary school/technical school education, and primary school education were lower than 
the overall score of public service satisfaction in Liaoning Province, followed by 66.54 points, 66.39 points, and 66.15 points. Among the first level indicator, the scores of groups with Master's degree or above in the living environment, public transportation, infrastructure, public security, culture and sports, compulsory education, pension service, employment service, social security and administrative convenience were higher than those of other education-level groups. The data showed that public service satisfaction evaluation increased with the improvement of public education, as shown in Figure 11.

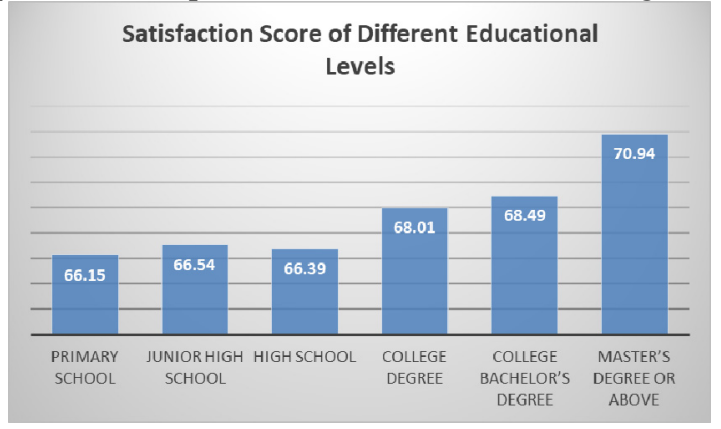

Figure 11. Public Satisfaction with Public Services by Different Education Background

\subsection{Monitoring of Social Public Service Quality by Different Income Groups}

People living in urban areas were more satisfied with government public services than those living in rural areas. Among the average monthly income groups of families in different cities, the satisfaction evaluation of public service in Liaoning Province was higher than that of the overall score of Liaoning Province, including $10,001 \sim 20,000$ yuan group, under 2,000 yuan group, $6,001 \sim 10,000$ yuan group and 2,001 4,000 yuan group, which were 69.34 points, 69.03 points, 68.40 points and 67.56 points respectively. Among the average monthly income groups of families in different rural areas, the score of 6,001-10,000 yuan group also showed higher characteristics than the overall score of public service social satisfaction evaluation in Liaoning Province, with the score of 68.13 points was also higher than that of Liaoning Province, as shown in Figure 12.

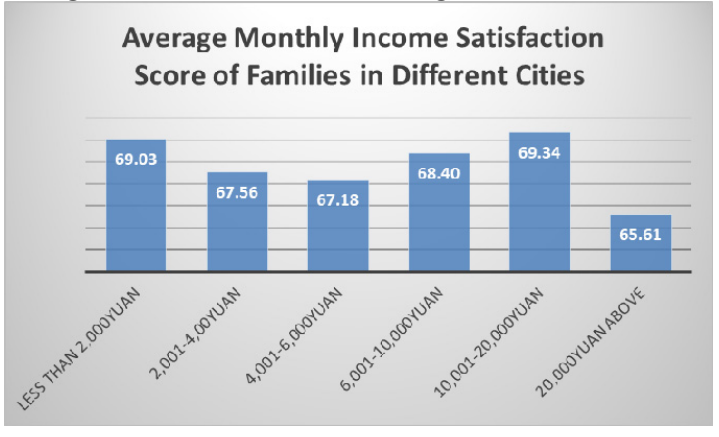

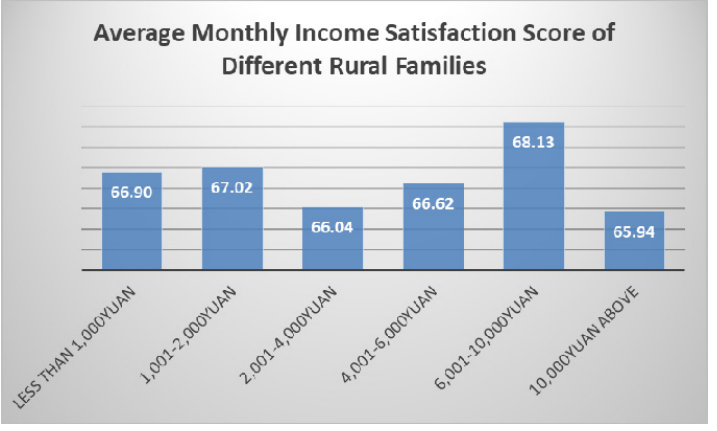

Figure 12. Public Satisfaction with Public Services in Urban and Rural Areas

Among the first level indicator, in this public services quality monitoring survey, the average monthly income of urban households group with 10,001 20,000 yuan was higher than other indicators in the public transportation, infrastructure, public security and social security; the average monthly income of rural households group with $6,001 \sim 10,000$ yuan was higher than that of other groups in living environment, public transport, culture and sports, compulsory education, administrative convenience and other indicators in this public service quality monitoring survey[9].

\section{Conclusions AND Suggestions}

Taking 14 cities in Liaoning Province as a survey, this paper collected data by means of face-to-face interviews on the streets and questionnaires, and conducted thirdparty quality monitoring and analysis in 11 public service areas, such as living environment, public transportation, infrastructure, medical and health care, public security, culture and sports, compulsory education, pension service, employment service, social security, administrative convenience[10]. The monitoring results showed that the public satisfaction with the services in five fields including social security, compulsory education, living environment, public security, and employment services was low. It was generally accepted that the attitude of public service personnel needed to be improved, public service facilities needed to be improved, the service level of subway, environment, social security and other service areas needed to be improved, the number of public services needed to be increased, and the work of government services needed to be improved. It is recommended that Liaoning province continues to participate in the satisfaction survey in depth, on the basis of data verification and analysis, timely understands the public's actual feelings and recognition of public service, makes scientific evaluation on the public's needs, optimizes decision-making, and ensures that satisfaction survey of public services becomes the thermometer for public demand.

\section{REFERENCES}

1. Wang Dianli and Song Xuezeng. An Empirical Study on Public Service Satisfaction Survey [J]. China Administration, 2009(6):72-77. 
2. Luo Xiaoguang and Zhang Hongyan. Evaluation dimension analysis of government service quality SERVQUAL [J]. Administrative Forum, 2008(3):3537.

3. Cui Guifang. On the Development of Government Service Quality Management [J]. Hubei Social Sciences, 2005(8):37-38.

4. Li Wenbin and Lai Linhui. Government Performance Satisfaction and Residents' Well-being: An Empirical Study in Guangdong Province [J]. China Administration, 2013(8):53-57.

5. Zhang E'e and Zhang Jing. Satisfaction Analysis of the Quality and Safety of Agricultural Products in Yongning County--Based on the Survey Data of 5 Township Residents in Yongning County [J]. Agricultural Science Research, 2017(3):92-96.
6. Robert B, Denhardt, Janet V. The New Public Service: Serving Rather Than Steering[J]. Public Administration Review, 2000,60(6):549-559.

7. Pfau, L.D (1989). Total Quality Management Gives Companies a Way to Enhance Position in Global Marketplace[J]. Industrial Engineering, 1989(21):1721.

8. Zweifel P, Zaborowsk C. Employment Service: Public or Private Public Choice[J]. Kluwer Academic Publishers, 1996.

9. Fretwell D, Goldberg S. Developing Effective Employment Services[J]. World Bank Discussion Papers, 1993.

10. Barbieria G: Do Public Employment Services Help People in Finding A Job[J]. An Evaluation of The Italian Case, 2001. 